\title{
Termine
}

http://doi.org/10.1515/bd-2018-0108

In der folgenden Terminzusammenstellung werden Fortbildungsseminare und ähnliche Fachveranstaltungen nachgewiesen, die zumindest einer eingeschränkten Öffentlichkeit zugänglich sind, außerdem Jahrestagungen und vergleichbare Anlässe umfassenderer Art. Hingegen können Termine nicht berücksichtigt werden, die ausschließlich lokaler Art oder für einen internen Kreis von Bedeutung sind, zumal in diesem Falle bei den Beteiligten in der Regel die Kenntnis des Termins vorausgesetzt werden kann. Soweit an anderer Stelle im Bibliotheksdienst nähere Informationen über einen bestimmten Termin abgedruckt sind, wird darauf verwiesen. Erstmalig aufgenommene Termine sind mit einem * gekennzeichnet.

\section{Dezember 2018}

3. „Verhaltensoriginelle“ Bibliotheksbenutzer? Anspruchsvolle Kundenkontakte und Konflikte professionell meistern, Delmenhorst (s. Heft 7, S. 589)

5. Fachtagung für Bibliotheksleiter/Innen in Öffentlichen Bibliotheken, Erfurt (s. Heft 2, S. 149)

12. Kreistreffen der Öffentlichen Bibliotheken und Landkreise. Sonneberg und Hildburghausen, Sonneberg (s. Heft 2, S. 152)

Januar 2019

*31.-1.2. Mit MALIS Zukunft gestalten - Konzepte und Strategien für die bibliothekarische Praxis, Köln (Ankündigung in diesem Heft) 